\title{
Laurent Polynomials and Superintegrable Maps
}

Andrew N.W. HONE

Institute of Mathematics, Statistics \& Actuarial Science, University of Kent, Canterbury CT2 7NF, UK

E-mail:anwh@kent.ac.uk

Received October 26, 2006; Published online February 07, 2007

Original article is available at http://www.emis.de/journals/SIGMA/2007/022/

\begin{abstract}
This article is dedicated to the memory of Vadim Kuznetsov, and begins with some of the author's recollections of him. Thereafter, a brief review of Somos sequences is provided, with particular focus being made on the integrable structure of Somos-4 recurrences, and on the Laurent property. Subsequently a family of fourth-order recurrences that share the Laurent property are considered, which are equivalent to Poisson maps in four dimensions. Two of these maps turn out to be superintegrable, and their iteration furnishes infinitely many solutions of some associated quartic Diophantine equations.
\end{abstract}

Key words: Laurent property; integrable maps; Somos sequences

2000 Mathematics Subject Classification: 11B37; 33E05; 37J35

\section{Introduction}

It is with considerable sadness that I begin to write this piece in memory of Vadim Kuznetsov, whose death came as a great shock to me. However, I do not wish to remain in melancholic mode, but rather I would like to recall some of my fondest and happiest memories of him.

While I was a PhD student in Edinburgh, I used to travel to Leeds every so often to attend the LMS workshops on integrable systems, and I'm sure I must have first met Vadim at one of these meetings. To begin with I remember his charming smile, as well as his relaxed way of asking penetrating mathematical questions. I also recall the great enthusiasm and energy with which he would give a seminar, and his clarity of presentation.

Shortly after graduating from Edinburgh, in September 1997 I went to Rome to take up my first postdoctoral position, working with Orlando Ragnisco in the Physics Department of Roma Tre. It was during this period that I had the privilege of getting to know Vadim a lot better. A few months after my arrival, he came to Rome to visit Orlando for a month, and the three of us ended up working together on a project that was suggested by Vadim, concerning Bäcklund transformations (BTs) for finite-dimensional integrable Hamiltonian systems. This turned out to be very fruitful, resulting in three joint publications [23, 24, 25].

Vadim's presence in Rome was immensely stimulating for me, because he succeeded in posing just the right question, at the precise moment when I had the necessary tools available to answer it. The specific problem that he first presented to me and Orlando was the construction of BTs for certain integrable classical mechanical systems corresponding to reduced Gaudin magnets. A particular concrete example of such a system was the case (ii) Hénon-Heiles system, an integrable system with two degrees of freedom. As it happened, in my PhD thesis I had already constructed an analogous BT for the non-autonomous case of this system, as well as deriving the explicit formula for the generating function of the canonical (contact) transformation in that case [22]. During my viva voce examination a few months earlier, Allan Fordy had

${ }^{\star}$ This paper is a contribution to the Vadim Kuznetsov Memorial Issue "Integrable Systems and Related Topics". The full collection is available at http://www.emis.de/journals/SIGMA/kuznetsov.html 
actually asked me whether the same sort of derivation could also be applied to the autonomous case, to produce a Poisson correspondence in the spirit of [16], and I could see no obvious obstruction. Thus it was that, when Vadim arrived in Rome, his vivid explanation of BTs, as well as his insistence that we should start constructing new ones, was all that I needed to work out the BT for the Hénon-Heiles system [23], and this soon revealed a similar algebraic structure underlying many other examples [24].

After he left Rome, I saw Vadim again in June 1998 at the conference Integrable Systems: Solutions and Transformations in Guardamar, Spain, where he came with his wife, Olga, and his son, Simon. We sat down together in the sunshine and completed some of the work on the second paper [24] while we were there. Subsequently, I saw Vadim sporadically at various meetings in Leeds and elsewhere, and we always found the time for a friendly chat about our lives and work. I particularly remember a very brief and enjoyable (but fiery) dispute that we had in Cambridge in 2001, while sitting together during an interlude between lectures in the Newton Institute. It boiled down to a minor difference in our points of view, which we respectively argued for without compromise, so that (having each seen the other's perspective) there was no love lost between us.

The rest of this article is concerned with a family of discrete dynamical systems (Poisson maps) in four dimensions, the first few of which are integrable, while the others are not. Before going into details, I should like to explain why I have chosen this topic. The work I did in my PhD was primarily concerned with integrable systems in the continuous setting (ordinary and partial differential equations), and it was not until Vadim's visit to Rome that I began to get actively interested in discrete systems. Ever since then, I have found the subject of discrete dynamics increasingly fascinating, and I shall always have Vadim to thank for inspiring me to look in this direction. Another interesting and unexpected property of the Poisson maps considered below is that their iterates are Laurent polynomials in the initial data; this is an instance of the Laurent phenomenon [14]. Vadim was an expert on special functions, and orthogonal polynomials in particular (for one of his many contributions in this area, see [35], for instance). However, most of the sequences of (Laurent) polynomials treated below satisfy nonlinear equations instead of linear ones.

The theory of discrete integrable maps has seen a great deal of activity in the past twenty years. The situation was much clarified by Veselov [57, 58, 59] who introduced integrable Lagrange correspondences - a natural discrete-time analogue of Liouville integrable continuous flows - which induce (generically multi-valued) shifts on the associated Liouville tori (see also [3]). Given a continuous integrable system, it is natural to seek a discretization of it that retains both the integrability and as many other properties as possible (e.g. Poisson structure, Lax pair, etc.). However, in general such a time-discretization will be implicit, and it will not preserve the same integrals as the original continuous system (see [52] for the state of the art in integrable discretizations). Building on results obtained for the Toda lattice by Pasquier and Gaudin [38], Kuznetsov and Sklyanin identified a special class of time-discretizations for integrable Hamiltonian systems which they referred to as BTs [32], by analogy with Bäcklund transformations for evolutionary PDEs.

In the setting of finite-dimensional systems with a Lax pair, BTs were identified as explicit Poisson maps which preserve the same set of integrals as the continuous flow that they discretize, and depend on a Bäcklund parameter $\lambda$ which satisfies a certain 'spectrality' property with respect to a conjugate variable $\mu$ (where $(\lambda, \mu)$ are the coordinates of a point on the spectral curve associated with the Lax pair). The viewpoint that I emphasized in [23, 24, 26] was that the systems being considered were reduced/stationary flows of the KdV hierarchy, whose BTs could be obtained by reduction from the Darboux-Bäcklund transformation for KdV (this is in the same vein as the dressing chain [60] - see also [63]), while the BTs in [25] were derived more directly. In Vadim's work with Pol Vanhaecke [34], all of the previously known 
examples were unified via an algebro-geometric approach, which explained the deeper meaning of BTs as discrete shifts on the (generalized) Jacobian of the associated spectral curve, thus identifying them as the discrete-time counterparts of algebraically completely integrable systems, as described in [56], for instance. While there has been subsequent work by Vadim and others on BTs in classical mechanics [8, 13, 39], a lot of the original motivation for studying them came from quantum integrable systems (Baxter's $Q$-operator). This idea has proved extremely effective (see e.g. [33, 35]), and will no doubt continue to bear fruit for a long time to come.

The last time I saw Vadim was in Leeds in April 2005, when he invited me to give one in the series of Quantum Computational seminars that he organized there ${ }^{1}$. At that time I spoke about Somos sequences, which are reviewed in the next section. In the evening after the seminar I went out for a very enjoyable dinner with Vadim and Olga, together with Oleg Chalykh and Sara Lombardo. I made an appointment to see Vadim in his office early the next morning, so that before my return home we had a good discussion about his recent work on the integrable dynamics of spin chains that arise in models of Fermi-Bose condensates [65] and BCS superconductors [66], and he described an unsolved problem concerning special solutions. This is how I remember him now: full of energy and always seeking to answer new questions.

\section{Somos sequences and the Laurent property}

The properties of integer sequences generated by linear recurrences have been the subject of a great deal of study in number theory, and nowadays they find applications in computer science and cryptography [12]. However, the theory of nonlinear recurrence sequences is still in its infancy. Clearly, a $k$ th-order nonlinear recurrence relation of the form

$$
x_{n+k}=F\left(x_{n}, x_{n+1}, \ldots, x_{n+k-1}\right)
$$

is just a particular sort of discrete dynamical system, so such recurrences can be considered as generating a special type of nonlinear dynamics. If we want (2.1) to generate sequences of integers, then choosing $F$ to be a polynomial with integer coefficients will certainly do the trick, but in general the corresponding map in $\mathbb{R}^{k}$ (or $\mathbb{C}^{k}$ ) will not have a unique inverse. Moreover, in that case such sequences generically exhibit double exponential growth i.e. $\log \left|x_{n}\right|$ grows exponentially with $n$. A simple example in this class is the quadratic map defined by the recurrence

$$
x_{n+1}=x_{n}^{2}+c
$$

with a parameter $c$, which is a prototypical model of chaos. However, note that the special cases $c=0,-2$ are exactly solvable [9], and in these cases one can also argue that (2.2) is integrable in the sense of admitting a commuting map (see [57] and references). The theory of linear recurrence sequences relies heavily on the fact that they are explicitly solvable. Thus it is natural to look for nonlinear recurrences that share this property, or that are integrable in a broader sense.

In the case that the map corresponding to (2.1) is invertible, one can also allow $F$ to be a rational function, thereby considering birational maps, but then it is no longer clear that integer sequences should result. However, it turns out that among those rational recurrences of the particular form

$$
x_{n+k} x_{n}=f\left(x_{n+1}, \ldots, x_{n+k-1}\right),
$$

\footnotetext{
${ }^{1}$ http://www.maths.leeds.ac.uk/ ${ }^{\sim}$ vadim/QCS.htm
} 
there is a very large class of recurrences that generate integer sequences from suitable initial data. One of the first known examples of this type is the Somos-4 recurrence

$$
x_{n+4} x_{n}=\alpha x_{n+3} x_{n+1}+\beta\left(x_{n+2}\right)^{2},
$$

which was found by Michael Somos when he was investigating the combinatorics of elliptic theta functions. Somos observed numerically that by taking the coefficients $\alpha=\beta=1$ and initial data $x_{0}=x_{1}=x_{2}=x_{3}=1$, the fourth-order recurrence (2.4) yields a sequence of integers [50], that is

$$
1,1,1,1,2,3,7,23,59,314,1529,8209,83313, \ldots
$$

Similarly he noticed that for the Somos- $k$ recurrences

$$
x_{n+k} x_{n}=\sum_{j=1}^{[k / 2]} \alpha_{j} x_{n+k-j} x_{n+j}
$$

with all coefficients $\alpha_{j}=1$, if all $k$ initial values are 1 then an integer sequence results for $k=5,6,7$, but denominators appear for $k=8$.

Various direct proofs that the terms of the sequence (2.5) are all integers were found at the beginning of the 1990s, when various other examples were found [17, 47], but a deeper understanding came from the realization that the recurrence (2.4) has the Laurent property: its iterates are all Laurent polynomials in the initial data (and in $\alpha, \beta$ ) with integer coefficients. To be more precise, the iterates of (2.4) satisfy $x_{n} \in \mathbb{Z}\left[x_{0}^{ \pm 1}, x_{1}^{ \pm 1}, x_{2}^{ \pm 1}, x_{3}^{ \pm 1}, \alpha, \beta\right]$ for all $n$, from which the integrality of the particular sequence (2.5) follows immediately. A little earlier, when Mills, Robbins and Rumsey made their study of the Dodgson condensation method for computing determinants [36] (which produced the famous alternating sign matrix conjecture [2]), they considered the recurrence

$$
D_{\ell, m, n+1} D_{\ell, m, n-1}=\alpha D_{\ell+1, m, n} D_{\ell-1, m, n}+\beta D_{\ell, m+1, n} D_{\ell, m-1, n}
$$

for $\alpha=1$ and observed that it produced Laurent polynomials in the initial data. The equation (2.7) thus became known within the algebraic combinatorics community, where it is referred to as the octahedron recurrence [43], while in the theory of integrable systems it is known as a particular form of the discrete Hirota equation [68] (the bilinear equation for the taufunction of discrete KP). The Somos-4 recurrence (2.4) is an ordinary difference reduction of the partial difference equation (2.7): it has been noted by Propp that if $x_{n}$ satisfies (2.4) then $D_{\ell, m, n}=x_{2 n+m}$ satisfies the discrete Hirota equation (see also [51] for another reduction).

Many more examples of this Laurent property have begun to emerge quite recently as an offshoot of the theory of cluster algebras due to Fomin and Zelevinsky (see [15] and references). The exchange relations in a cluster algebra of rank $k$ are typified by a recurrence of the form

$$
x_{n+k} x_{n}=c_{1} M_{1}\left(x_{n+1}, \ldots, x_{n+k-1}\right)+c_{2} M_{2}\left(x_{n+1}, \ldots, x_{n+k-1}\right)
$$

for suitable monomials $M_{j}$ and coefficients $c_{j}$, which is a special case of (2.3). In [14], the general machinery of cluster algebras was shown to be very effective in proving the Laurent property for a wide variety of recurrences, mostly (but not all) of the form (2.8). In particular, Fomin and Zelevinsky there gave the first proof of the Laurent property for the octahedron (discrete Hirota) recurrence (2.7). Subsequently, Speyer has developed a combinatorial model to prove more detailed properties of the Laurent polynomials generated by this recurrence - in particular, that all the coefficients are 1 [51]. 
So far we have discussed the integrality of the sequence (2.5), but not the integrability of the Somos-4 recurrence. Taking $\left(x_{0}, x_{1}, x_{2}, x_{3}\right)$ as coordinates, the map $\mathbb{C}^{4} \rightarrow \mathbb{C}^{4}$ corresponding to (2.4) preserves the degenerate Poisson bracket defined by

$$
\left\{x_{m}, x_{n}\right\}_{2}=(n-m) x_{m} x_{n}
$$

which has Casimirs

$$
u_{n}=\frac{x_{n-1} x_{n+1}}{\left(x_{n}\right)^{2}}, \quad n=1,2 .
$$

This bracket is of the 'log-canonical' type that has previously been found in the context of cluster algebras [18]; it is natural to consider it as a Poisson bracket on the field of rational functions $\mathbb{C}\left(x_{0}, x_{1}, x_{2}, x_{3}\right)$. (The reason for the subscript 2 on the bracket will become apparent in the next section.) The set of solutions of (2.4) is invariant under the two-parameter Abelian group of gauge transformations generated by

$$
x_{n} \mapsto A x_{n}, \quad x_{n} \mapsto B^{n} x_{n}, \quad A, B \in \mathbb{C}^{*} .
$$

The Hamiltonian vector fields corresponding to these transformations are respectively generated by the rational monomials

$$
K_{1}=\frac{x_{1}}{x_{2}}, \quad K_{2}=\frac{\left(x_{1}\right)^{2}}{x_{2}}
$$

which satisfy

$$
\left\{K_{1}, x_{n}\right\}_{2}=K_{1} x_{n}, \quad\left\{K_{2}, x_{n}\right\}_{2}=n K_{2} x_{n}, \quad\left\{K_{1}, K_{2}\right\}_{2}=K_{1} K_{2} .
$$

In fact, the most interesting part of the dynamics generated by (2.4) takes place in the plane spanned by the Casimirs $u_{1}, u_{2}$ for the bracket $\{\cdot, \cdot\}_{2}$. If we take the definition (2.10) to hold for all $n$, then the quantities $u_{n}$ are clearly invariant under the gauge transformations (2.11), and satisfy the second-order recurrence

$$
u_{n+2}=\frac{\alpha u_{n+1}+\beta}{u_{n}\left(u_{n+1}\right)^{2}} .
$$

(So the fourth-order equation (2.4) is the Hirota bilinearization of (2.13), which is a second-order ordinary difference equation.) By taking $\left(u_{1}, u_{2}\right)$ as coordinates in $\mathbb{C}^{2}$, this corresponds to the rational map of the plane given by

$$
\left(\begin{array}{l}
u_{1} \\
u_{2}
\end{array}\right) \mapsto\left(\begin{array}{c}
u_{2} \\
\left(\alpha u_{2}+\beta\right) /\left(u_{1}\left(u_{2}\right)^{2}\right)
\end{array}\right)
$$

which preserves the Poisson bracket

$$
\left\{u_{n}, u_{n+1}\right\}=u_{n} u_{n+1},
$$

or equivalently the symplectic form

$$
\omega_{n}=\left(u_{n} u_{n+1}\right)^{-1} \mathrm{~d} u_{n} \wedge \mathrm{d} u_{n+1}
$$

such that $\omega_{n+1}=\omega_{n}$. Furthermore, this has the conserved quantity

$$
J=u_{n} u_{n+1}+\alpha\left(\frac{1}{u_{n}}+\frac{1}{u_{n+1}}\right)+\frac{\beta}{u_{n} u_{n+1}},
$$


which defines a quartic curve

$$
\zeta^{2} \eta^{2}-J \zeta \eta+\alpha(\zeta+\eta)+\beta=0
$$

of genus one. Hence we see that (2.13) produces a Liouville integrable system with one degree of freedom, and the curve (2.17) itself defines the two-valued correspondence $u_{n} \mapsto u_{n \pm 1}$, which is a particular case of the Euler-Chasles correspondence (see $[57,58,59]$ ).

Upon uniformizing the elliptic quartic we find that the explicit solution to (2.13) is given by

$$
u_{n}=\wp(z)-\wp\left(z_{0}+n z\right)
$$

in terms of the Weierstrass $\wp$ function for the elliptic curve

$$
E: \quad Y^{2}=4 X^{3}-g_{2} X-g_{3},
$$

with $g_{2}=12 \lambda^{2}-2 J, g_{3}=4 \lambda^{3}-g_{2} \lambda-\alpha, \lambda=\left(J^{2} / 4-\beta\right) /(3 \alpha)$, and $z_{0}, z \in \mathbb{C} / \Lambda=\operatorname{Jac}(E)$ are given by elliptic integrals obtained from inversion of the relations $\wp(z)=\lambda, \wp\left(z_{0}\right)=\lambda-u_{0}$. The coefficients $\alpha, \beta$ and also $J$ are given as elliptic functions of $z$ by $\alpha=\wp^{\prime}(z)^{2}, \beta=\wp^{\prime}(z)^{2}(\wp(2 z)-$ $\wp(z)), J=\wp^{\prime \prime}(z)$.

From this it follows [27] that the solution to the initial value problem for the Somos-4 recurrence (2.4) can be written in terms of the Weierstrass sigma function as

$$
x_{n}=A B^{n} \frac{\sigma\left(z_{0}+n z\right)}{\sigma(z)^{n^{2}}}
$$

for suitable $A, B$. There is an analogous formula for the general solution of the Somos-5 recurrence [28], which has an additional dependence on the parity of $n$.

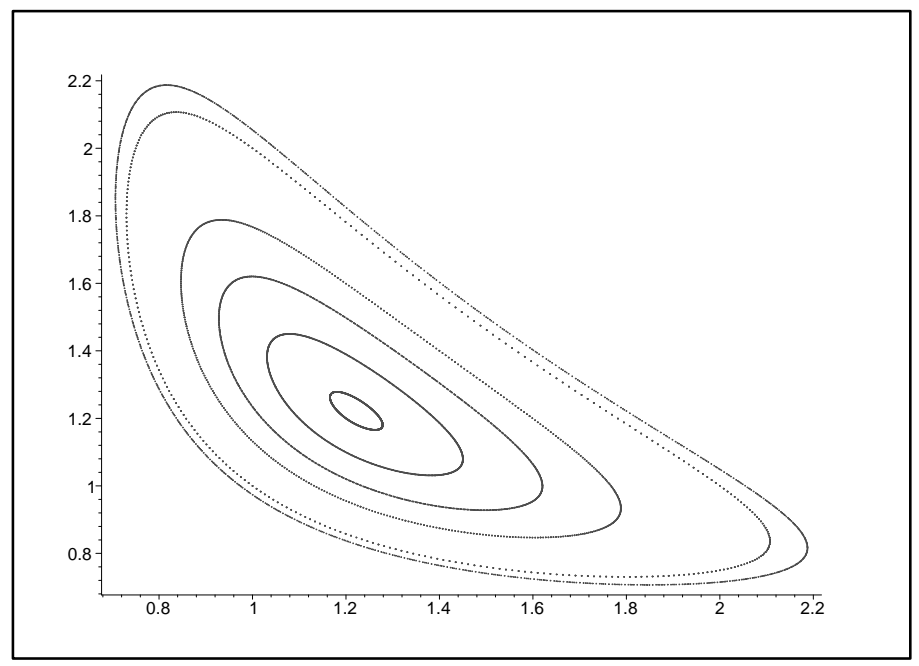

Figure 1. A family of orbits for the nonlinear recurrence (2.13) associated with Somos-4.

The map defined by (2.13) is a very simple example of the QRT family [45]. It has a $2 \times 2$ discrete Lax pair given by

$$
\mathbf{L}_{n} \Psi_{n}=\nu \Psi_{n}, \quad \Psi_{n+1}=\mathbf{M}_{n} \Psi_{n},
$$

where

$$
\mathbf{L}_{n}(v)=\left(\begin{array}{cc}
u_{n} u_{n+1} & -\alpha \\
v-u_{n}-u_{n+1} & \alpha\left(1 / u_{n}+1 / u_{n+1}\right)+\beta /\left(u_{n} u_{n+1}\right)
\end{array}\right),
$$




$$
\mathbf{M}_{n}(v)=\left(\begin{array}{cc}
0 & -\alpha \\
v-u_{n}-u_{n+1} & \alpha / u_{n}
\end{array}\right) .
$$

The equation (2.13) arises as the compatibility condition $\mathbf{L}_{n+1} \mathbf{M}_{n}=\mathbf{M}_{n} \mathbf{L}_{n}$ for the system (2.21), and the associated spectral curve is given by

$$
\operatorname{det}\left(\mathbf{L}_{n}(v)-\nu \mathbf{1}\right)=\nu^{2}-J \nu+\alpha v+\beta=0,
$$

where $J$ is the conserved quantity given by (2.16). From the formulae $\nu=\zeta \eta, v=\zeta+\eta$ the elliptic quartic curve (2.17) is seen to be a ramified double cover of this rational (genus zero) spectral curve.

It is clear from the above considerations that the dynamics of (2.13) corresponds to a sequence of points $P_{0}+n P$ on the elliptic curve $E$ given by (2.19), or to the equivalent discrete linear flow $z_{0}+n z$ on its Jacobian, and in that sense (as was noted in [27]) it is the same as the underlying dynamics of the BT for the one-particle Garnier system constructed in [24], or that of the BT for the $g=1$ odd Mumford system as in [34]. However, while one can make changes of variables between (2.13) and each of the latter two BTs, they are not canonical transformations, because the Poisson bracket (2.14) is incompatible with the Poisson structures of either of these BTs. Nevertheless, just as for the BTs, the recurrence (2.13) is a discretization of a continuous time integrable system with the same Poisson structure and conserved quantities (in this case, only one of them), namely the flow in the plane with Hamiltonian $J$ defined by (2.16) with $n=1$, i.e.

$$
\begin{aligned}
& \frac{\mathrm{d} u_{1}}{\mathrm{~d} t}=\left\{J, u_{1}\right\}=\left(\alpha u_{1}+\beta\right) / u_{2}-\left(u_{1}\right)^{2} u_{2}, \\
& \frac{\mathrm{d} u_{2}}{\mathrm{~d} t}=\left\{J, u_{2}\right\}=-\left(\alpha u_{2}+\beta\right) / u_{1}+\left(u_{2}\right)^{2} u_{1} .
\end{aligned}
$$

From the same uniformization of the quartic (2.17) as before, the solution of the system (2.23) can be written down as

$$
u_{1}(t)=\wp(z)-\wp\left(\tilde{z}_{0}+\wp^{\prime}(z) t\right), \quad u_{2}(t)=\wp(z)-\wp\left(\tilde{z}_{0}+z+\wp^{\prime}(z) t\right),
$$

and upon comparison with (2.18) it can be seen directly how the discrete flow interpolates the continuous one (cf. Fig. 1).

The construction of a sequence of points $P_{0}+n P$ on elliptic curve $E$ from a Somos- 4 or Somos-5 sequence was previously understood in unpublished work of several number theorists ${ }^{2}-$ see the discussion of Zagier [67], and the results of Elkies quoted in [4]. The algebraic part of the construction is described in the thesis of Swart [53] (who also mentions unpublished results of Nelson Stephens), and van der Poorten has recently presented another construction based on the continued fraction expansion of the square root of a quartic [41]. In fact, Somos-4 sequences have an ancestor from the 1940s, in Morgan Ward's work on elliptic divisibility sequences (EDS), which just correspond to multiples of a point $n P \in E[61,62]$ i.e. this is the special case $P_{0}=\infty$, so that $z_{0}=0$, with the further requirement that $A=B=1$ in (2.20). The iterates of an EDS, which are generated by (2.4) with coefficients $\alpha=\left(x_{2}\right)^{2}, \beta=-x_{1} x_{3}$ and integer initial data $x_{1}=1, x_{2}, x_{3}, x_{4} \in \mathbb{Z}$ with $x_{2} \mid x_{4}$, satisfy the divisibility property $x_{m} \mid x_{n}$ whenever $m \mid n$, and correspond to values of the division polynomials of the curve (for a description of these see Exercise 3.7 in [48]). In this sense, an EDS generalizes properties of certain linear recurrence sequences. For example, the Fibonacci numbers are generated by the recurrence $F_{n+1}=F_{n}+F_{n-1}$ with initial values $F_{0}=1, F_{1}=1$, and form a divisibility sequence. Moreover, the even index terms $x_{n}=F_{2 n}$ form a divisibility sequence (so $F_{2 m} \mid F_{2 n}$ whenever $m \mid n$ ) and also satisfy the Somos-4 recurrence

$$
F_{2 n+4} F_{2 n-4}=9 F_{2 n+2} F_{2 n-2}-8\left(F_{2 n}\right)^{2},
$$

\footnotetext{
${ }^{2}$ For further references see http://www.math.wisc.edu/ propp/somos.html.
} 
which corresponds to a degenerate case of the curve (2.19) where the discriminant vanishes, so $g_{2}^{3}-27 g_{3}^{2}=0$ and the formula (2.20) degenerates to an expression in terms of the hyperbolic sine.

The arithmetical properties of EDS and Somos sequences - in particular the distribution of primes therein - are a subject of current interest [10, 11, 49]. Some of these properties are discussed in the book [12] (see section 1.1.20, for instance), where it is suggested that such bilinear recurrences should be suitable generalizations of linear ones, with many analogous features. Based on the appearance of higher-order Somos recurrences in the work of Cantor on the analogues of division polynomials for hyperelliptic curves [6] (see also [40] for analytic formulae), it was conjectured in [27] that every Somos- $k$ sequence should correspond to a discrete linear flow on the Jacobian of such a curve (with an associated discrete integrable system), and the plausibility of this conjecture was justified by a naïve counting argument. However, on Propp's bilinear forum [44], Elkies had already given a more detailed argument to the contrary, based on a proposed theta function formula for the terms of such sequences, which indicated that while the general Somos-6 and Somos-7 sequence could be described by such a formula in genus two, the general Somos- $k$ for $k \geq 8$ could not. Thus in this setting the absence of the Laurent property appears to coincide with the absence of algebraic integrability.

Nevertheless, in [1] it was shown that a particular family of solutions of Somos- 8 recurrences can be described in terms of the Kleinian sigma function for a genus two curve (which is equivalent to an expression in theta functions), and these solutions are related to the BT for the Hénon-Heiles system that was found in $[23,24]$. The author has also found that the Somos-6 and Somos-7 recurrences correspond to a rational map in $\mathbb{C}^{4}$ with two independent conserved quantities, and there is a similar expression for the solutions in terms of genus two sigma functions. For instance, letting $\sigma$ denote the genus two Kleinian sigma function (see e.g. [5] for the definition), associated with a curve given by the affine equation $y^{2}=4 x^{5}+c_{3} x^{3}+\cdots+c_{0}$ with period lattice $\Lambda$, the expression

$$
x_{n}=A B^{n} \frac{\sigma\left(\mathbf{v}_{0}+n \mathbf{v}\right)}{\sigma(\mathbf{v})^{n^{2}}},
$$

where $A, B \in \mathbb{C}^{*}, \mathbf{v}_{0}, \mathbf{v} \in \mathbb{C}^{2} \bmod \Lambda$, satisfies a Somos-6 recurrence provided that $\mathbf{v}$ is constrained according to

$$
\left|\begin{array}{ccc}
1 & 1 & 1 \\
\wp_{12}(\mathbf{v}) & \wp_{12}(2 \mathbf{v}) & \wp_{12}(3 \mathbf{v}) \\
\wp_{22}(\mathbf{v}) & \wp_{22}(2 \mathbf{v}) & \wp_{22}(3 \mathbf{v})
\end{array}\right|=0
$$

where $\wp_{j k}(\mathbf{v})=-\partial_{j} \partial_{k} \log \sigma(\mathbf{v})$ are the corresponding Kleinian $\wp$ functions. In the case of generic $\mathbf{v}$, if this constraint does not hold, then $x_{n}$ given by (2.24) satisfies a Somos- 8 recurrence instead. The full details will be presented elsewhere [31].

Before moving onto other examples in the next section, we should mention one more feature of the Somos-4 recurrence, namely the fact that it generates solutions of a quartic Diophantine equation in four variables. If we rewrite the formula (2.16) for the conserved quantity $J$ in terms of the original variables $x_{n}$, we obtain the equation

$$
\begin{aligned}
& \left(x_{n-1}\right)^{2}\left(x_{n+2}\right)^{2}+\alpha\left(x_{n-1}\left(x_{n+1}\right)^{3}+\left(x_{n}\right)^{3} x_{n+2}\right)+\beta\left(x_{n}\right)^{2}\left(x_{n+1}\right)^{2} \\
& \quad=J x_{n-1} x_{n} x_{n+1} x_{n+2} .
\end{aligned}
$$

If we have coefficients $\alpha, \beta \in \mathbb{Z}$ (or in $\mathbb{Q}$ ), and if the Somos-4 recurrence (2.4) with a set of integer initial data $\left(x_{0}, x_{1}, x_{2}, x_{3}\right)$ generates a non-periodic sequence of iterates satisfying $x_{n} \in \mathbb{Z}$ for all $n$, then there are infinitely many quadruples of integers $\left(x_{n-1}, x_{n}, x_{n+1}, x_{n+2}\right)$ 
that are solutions of the quartic Diophantine equation (2.25). (Note that in this case, as long as all the integer initial data are non-zero, then the coefficient $J$ which appears in (2.25) is uniquely determined, and $J \in \mathbb{Q}$.) This can be seen as a particular instance of a general feature shared by all recurrences that both have the Laurent property and possess a rational invariant: generically, the orbit of suitable initial data will generate infinitely many solutions of an associated Diophantine equation.

Diophantine Laurentness Lemma. Suppose that a kth-order rational recurrence of the form (2.1) has coefficients in $\mathbb{Q}[\mathbf{c}]$ (for some set of parameters $\mathbf{c}$ ) and has the Laurent property, i.e. $x_{n} \in \mathbb{Z}\left[x_{0}^{ \pm 1}, x_{1}^{ \pm 1}, \ldots, x_{k-1}^{ \pm 1}, \mathbf{c}\right]$ for all $n$. Suppose further that this recurrence also has a rational conserved quantity given by

$$
K=\frac{f_{1}\left(x_{n}, x_{n+1}, \ldots, x_{n+k-1}, \mathbf{c}\right)}{f_{2}\left(x_{n}, x_{n+1}, \ldots, x_{n+k-1}, \mathbf{c}\right)}
$$

for $f_{1}, f_{2} \in \mathbb{Z}\left[x_{n}, x_{n+1}, \ldots, x_{n+k-1}, \mathbf{c}\right]$. If $f_{2} \neq 0$ for some fixed integer values of $\mathbf{c}$ and initial data $x_{j}=1$ or -1 for $j=0, \ldots, k-1$, then the value of $K \in \mathbb{Q}$ is fixed, and the recurrence generates infinitely many integer solutions of the Diophantine equation

$$
f_{1}\left(x_{n}, x_{n+1}, \ldots, x_{n+k-1}, \mathbf{c}\right)=K f_{2}\left(x_{n}, x_{n+1}, \ldots, x_{n+k-1}, \mathbf{c}\right)
$$

as long as the corresponding orbit is not periodic.

The integer sequence (2.5) provides a concrete example of the above result: setting $\alpha=\beta=1$, the initial data $1,1,1,1$ yield the value $J=4$ in (2.25), and for $n \geq 0$ any four adjacent terms of this increasing sequence provide a distinct solution of the equation. In fact, in [54] it is proved that the iterates of the Somos- 4 recurrence satisfy the stronger property that $x_{n} \in$ $\mathbb{Z}\left[x_{0}^{ \pm 1}, x_{1}, x_{2}, x_{3}, \alpha, \beta,\left(\alpha^{2}+\beta J\right)\right]$ for $n \geq 0$, which yields a broader set of sufficient criteria for integer sequences to be generated. In the next section we will see analogous results for some other recurrences.

\section{A fourth-order family}

A generalization of (2.4), that retains the Laurent property, is the family of fourth-order recurrences

$$
x_{n+2} x_{n-2}=x_{n+1}^{a} x_{n-1}^{b}+x_{n}^{c},
$$

where the exponents $a, b, c$ are positive integers. These generalized Somos- 4 recurrences were first described in print by David Gale [17], who noted that they all generate integer sequences from the initial values $x_{0}=x_{1}=x_{2}=x_{3}=1$. Among various examples covered in [14], Fomin and Zelevinksy subsequently proved that each of these recurrences has the Laurent property. However, in contrast to the integrable structure of the original Somos-4 recurrence, most of these examples do not seem to correspond to completely integrable systems.

Below we shall not present an analysis of the complete family (3.1), but rather we focus on the special sub-family of recurrences defined by $a=b=1$, with $c \in \mathbb{N}$. In this case, it will be convenient to introduce a parameter $\beta$ as the coefficient of the second term on the right hand side; although this can always be removed by rescaling $x_{n}$, its inclusion preserves the Laurent property (while inserting another coefficient $\alpha$ in front of the $x_{n+3} x_{n+1}$ term does not, unless $c=2$ ). These recurrences also satisfy the singularity confinement test that was proposed in [19] as an analogue of the Painlevé test for discrete equations: if an apparent singularity is reached (in this case, corresponding to the situation that one of the iterates vanishes), then it is always possible to analytically continue through it. 
Proposition 1. For each $c \in \mathbb{N}$ the recurrence

$$
x_{n+4} x_{n}=x_{n+3} x_{n+1}+\beta x_{n+2}^{c},
$$

which corresponds to the iteration of the rational map

$$
\left(\begin{array}{c}
x_{0} \\
x_{1} \\
x_{2} \\
x_{3}
\end{array}\right) \mapsto\left(\begin{array}{c}
x_{1} \\
x_{2} \\
x_{3} \\
\left(x_{1} x_{3}+\beta x_{2}^{c}\right) / x_{0}
\end{array}\right)
$$

has the Laurent property in the sense that $x_{n} \in \mathbb{Z}\left[x_{0}^{ \pm 1}, x_{1}^{ \pm 1}, x_{2}^{ \pm 1}, x_{3}^{ \pm 1}, \beta\right]$ for all $n \in \mathbb{Z}$, and also satisfies the singularity confinement test. Furthermore, (3.3) is a Poisson map with respect to the log-canonical Poisson bracket $\{\cdot, \cdot\}_{c}$ defined by

$$
\begin{array}{lll}
\left\{x_{0}, x_{1}\right\}_{c}=x_{0} x_{1}, & \left\{x_{0}, x_{2}\right\}_{c}=c x_{0} x_{2}, \quad\left\{x_{0}, x_{3}\right\}_{c}=(c+1) x_{0} x_{3}, \\
\left\{x_{1}, x_{2}\right\}_{c}=x_{1} x_{2}, & \left\{x_{1}, x_{3}\right\}_{c}=c x_{1} x_{3}, \quad\left\{x_{2}, x_{3}\right\}_{c}=x_{2} x_{3},
\end{array}
$$

which is nondegenerate for $c \neq 2$.

Proof. The recurrence (3.2) is of the cluster algebra type, so the Laurent property can be proved by the methods of [14], where the details for the complete family (3.1) are presented. However, here it is convenient to sketch a direct proof by induction, as this will have singularity confinement as an immediate corollary. The inductive hypothesis is that any four adjacent iterates $x_{k}, x_{k+1}, x_{k+2}, x_{k+3}$ for $0 \leq k \leq n+4$ are coprime elements of the unique factorization domain $\mathcal{R}=\mathbb{Z}\left[x_{0}^{ \pm 1}, x_{1}^{ \pm 1}, x_{2}^{ \pm 1}, x_{3}^{ \pm 1}, \beta\right]$, which has units $\pm x_{0}^{\ell_{0}}, x_{1}^{\ell_{1}}, x_{2}^{\ell_{2}} x_{3}^{\ell_{3}}$ for $\ell_{j} \in \mathbb{Z}$. Working $\bmod x_{n+4}$, the congruences $x_{n+5} \equiv \beta x_{n+3}^{c} x_{n+1}^{-1}, x_{n+6} \equiv \beta x_{n+3}^{c+1} x_{n+2}^{-1} x_{n+1}^{-1}, x_{n+7} \equiv \beta^{c+1} x_{n+3}^{c^{2}-1} x_{n+1}^{-c}$ all hold, so that

$$
x_{n+8} x_{n+4} \equiv x_{n+3}^{c^{2}+c-1} x_{n+2}^{-c} x_{n+1}^{-c-1}\left(x_{n+3} x_{n+1}+\beta x_{n+2}^{c}\right) \equiv 0,
$$

since the bracketed expression in the middle is just $x_{n+4} x_{n}$ by (3.2). This proves the inductive step that $x_{n+8} \in \mathcal{R}$, and it is easy to see from (3.2) that this element is coprime to $x_{n+5}$, $x_{n+6}, x_{n+7}$; the base of the induction is trivial. This argument also demonstrates singularity confinement: if we have $x_{n+4}=\left(x_{n+3} x_{n+1}+\beta x_{n+2}^{c}\right) / x_{n}=\epsilon \rightarrow 0$ for some $n$, so that $x_{n+8}$ is potentially singular, then the preceding calculation shows that $x_{n+8} x_{n+4}=O(\epsilon)$ and hence $x_{n+8}=O(1)$ as $\epsilon \rightarrow 0$, so that the singularity is confined. The Poissonicity of the map (3.3) is checked by a direct calculation, and in the coordinates $y_{n}=\log x_{n}$ the Poisson tensor for the bracket $\{\cdot, \cdot\}_{c}$ is constant and has determinant $(c-2)^{2}(c+1)^{2}$. Thus for $c \in \mathbb{N}$ it is nondegenerate unless $c=2$, which gives the previously mentioned bracket (2.9) preserved by the Somos-4 recurrence.

Remark 1. In the case of arbitrary parameters $a=b$ and $c$, each of the recurrences (3.2) admits a log-canonical Poisson bracket that generalizes $\{\cdot, \cdot\}_{c}$, but there is no such bracket for $a \neq b$.

Corollary 1. For each $c$ the two-form

$$
\begin{aligned}
\omega= & \left(\frac{\mathrm{d} x_{0} \wedge \mathrm{d} x_{1}}{x_{0} x_{1}}+\frac{\mathrm{d} x_{0} \wedge \mathrm{d} x_{3}}{x_{0} x_{3}}+\frac{\mathrm{d} x_{2} \wedge \mathrm{d} x_{3}}{x_{2} x_{3}}\right) \\
& -c\left(\frac{\mathrm{d} x_{0} \wedge \mathrm{d} x_{2}}{x_{0} x_{2}}+\frac{\mathrm{d} x_{1} \wedge \mathrm{d} x_{3}}{x_{1} x_{3}}\right)+(c+1) \frac{\mathrm{d} x_{1} \wedge \mathrm{d} x_{2}}{x_{1} x_{2}},
\end{aligned}
$$

is preserved by the map (3.3), and this is symplectic for $c \neq 2$. When $c=2$ this two-form is degenerate, being the pullback of the two-form $\omega_{1}$ in (2.15) under the transformation

$$
\left(x_{0}, x_{1}, x_{2}, x_{3}\right) \mapsto\left(u_{1}, u_{2}\right)=\left(x_{0} x_{2} /\left(x_{1}\right)^{2}, x_{1} x_{3} /\left(x_{2}\right)^{2}\right) .
$$


We should now like to assert that the recurrences (3.2) do not correspond to algebraically completely integrable systems when $c \geq 3$, based on the fact that in that case they have nonzero algebraic entropy. Recall that for a rational map, the algebraic entropy is defined as $\lim _{n \rightarrow \infty}\left(\log d_{n}\right) / n$, where $d_{n}$ is the degree of the $n$th iterate of the map [21]. Usually finding this limit requires extensive calculations of the corresponding sequence of rational functions of the initial data, or of the iterates of the projectivized form of the map. However, in this case we can exploit the fact that these recurrences generate Laurent polynomials, as well as the rescaling $x_{n} \rightarrow \beta^{-1 /(c-2)} x_{n}$ (which for $c \neq 2$ is equivalent to setting $\beta=1$ in (3.2)), to argue that the degrees of the iterates as polynomials in the coefficient $\beta$ gives a suitable measure of the entropy.

Proposition 2. For $c \in \mathbb{N}$, the nth iterate of (3.2) is a polynomial in $\beta$ of degree $d_{n}$, as well as a Laurent polynomial in the initial data, where $d_{n}$ satisfies the recurrence

$$
d_{n+2}+d_{n-2}=\max \left\{c d_{n}+1, d_{n+1}+d_{n-1}\right\}
$$

for $n \geq 2$, with initial data $d_{0}=d_{1}=d_{2}=d_{3}=0$. The algebraic entropy of the recurrence is zero for $c=0,1,2$, while for $c \geq 3$ it is given by

$$
\lim _{n \rightarrow \infty}\left(\log d_{n}\right) / n=\frac{1}{2} \log \left(\frac{c+\sqrt{c^{2}-4}}{2}\right) .
$$

Remark 2. The full analysis of the 'tropical' (or piecewise-linear) recurrence (3.6) is somewhat involved, and is omitted here, but we can mention that the determination of the value (3.7) for the algebraic entropy follows from the fact that when $c \geq 3$ the degrees just satisfy the linear recurrence

$$
d_{n+2}+d_{n-2}=c d_{n}+1
$$

when $n \geq 6$, and hence they grow exponentially with $n$. For $c=0,1$ the growth of $d_{n}$ is linear in $n$, while for $c=2$ it is quadratic in $n$ (corresponding to the quadratic growth of logarithmic heights on elliptic curves [48]). Very similar analysis shows that for $c \geq 3$ the recurrences (3.1) fail Halburd's Diophantine integrability criterion [20], which requires that the logarithmic heights of all rational-valued iterates should grow no faster than polynomial in $n$. For instance, with initial data $x_{0}=x_{1}=x_{2}=x_{3}=1$ each recurrence generates polynomials in $\mathbb{Z}[\beta]$, and upon evaluating these at generic values of $\beta \in \mathbb{Q}$ it can be demonstrated that the logarithmic heights of these numbers grow like the degrees $d_{n}$.

Having isolated the cases $c=0,1,2$, we shall describe their integrable structure (in descending order). The case $c=2$ is the original Somos- 4 recurrence (2.4) that was treated in the previous section, so we proceed with $c=1$.

Theorem 1. The map (3.3) for $c=1$ is superintegrable, in the sense that it has three independent conserved quantities $\mathcal{J}_{k}, k=1,2,3$, which satisfy

$$
\left\{\mathcal{J}_{1}, \mathcal{J}_{2}\right\}_{1}=0=\left\{\mathcal{J}_{1}, \mathcal{J}_{3}\right\}_{1}
$$

where

$$
\mathcal{J}_{1}=\mathcal{C}_{0} \mathcal{C}_{1} \mathcal{C}_{2}-\mathcal{C}_{0}^{2}-\mathcal{C}_{1}^{2}-\mathcal{C}_{2}^{2}+2, \quad \mathcal{J}_{2}=\mathcal{C}_{0}+\mathcal{C}_{1}+\mathcal{C}_{2}, \quad \mathcal{J}_{3}=\mathcal{C}_{0} \mathcal{C}_{1} \mathcal{C}_{2}
$$

with

$$
\mathcal{C}_{0}=\frac{x_{0} x_{3}+x_{1}^{2}+x_{2}^{2}}{x_{1} x_{2}}, \quad \mathcal{C}_{1}=\frac{x_{0} x_{3}^{2}+x_{1}^{2} x_{3}+x_{0} x_{2}^{2}+\beta x_{1} x_{2}}{x_{0} x_{2} x_{3}}
$$




$$
\mathcal{C}_{2}=\frac{x_{0}^{2} x_{3}+x_{1}^{2} x_{3}+x_{0} x_{2}^{2}+\beta x_{1} x_{2}}{x_{0} x_{1} x_{3}} .
$$

The iterates of the corresponding recurrence

$$
x_{n+2} x_{n-2}=x_{n+1} x_{n-1}+\beta x_{n},
$$

also satisfy the ninth-order linear recurrence

$$
x_{n+9}-\left(\mathcal{J}_{1}+1\right)\left(x_{n+6}-x_{n+3}\right)-x_{n}=0,
$$

and the solution of the initial value problem for (3.11) has the form

$$
x_{3 n+j}=\mathcal{A}_{j} T_{n}\left(\mathcal{J}_{1} / 2\right)+\mathcal{B}_{j} U_{n}\left(\mathcal{J}_{1} / 2\right)+\beta \mathcal{C}_{j} /\left(\mathcal{J}_{1}-2\right), \quad j=0,1,2,
$$

where $T_{n}$ and $U_{n}$ are the Chebyshev polynomials of the first and second kind respectively, and the coefficients $\mathcal{A}_{j}, \mathcal{B}_{j}$ are given by

$$
\mathcal{A}_{j}=2 x_{j}-\frac{2 x_{j+3}}{\mathcal{J}_{1}}-\frac{2 \beta \mathcal{C}_{j}\left(\mathcal{J}_{1}-1\right)}{\mathcal{J}_{1}\left(\mathcal{J}_{1}-2\right)}, \quad \mathcal{B}_{j}=-x_{j}+\frac{2 x_{j+3}+\beta \mathcal{C}_{j}}{\mathcal{J}_{1}}
$$

for $j=0,1,2$.

Proof. The proof of the above result is only sketched here, as further details will be presented elsewhere [31]. The main observation is that the recurrence (3.11) is linearizable, in the sense that the iterates satisfy the higher-order linear recurrence (3.12) for a suitable $\mathcal{J}_{1}$. (In the case $\beta=1$, the nonlinear recurrence was originally considered by Dana Scott [17], who found that an integer sequence was generated from the initial data $x_{0}=x_{1}=x_{2}=x_{3}=1$; in that case the linear recurrence $(3.12)$ is satisfied with $\mathcal{J}_{1}=9$.) In general one can take (3.12) as the definition of $\mathcal{J}_{1}$, and use (3.11) to back-substitute and rewrite it in terms of four adjacent iterates

$$
\left(x_{n}, x_{n+1}, x_{n+2}, x_{n+3}\right)=(p, q, r, s),
$$

as

$$
\mathcal{J}_{1}=\frac{\left(p^{2}+s^{2}\right) q r+\beta(p+s)\left(q^{2}+r^{2}+p s\right)+\beta^{2} q r}{p q r s},
$$

which is found to be invariant with $n$, and defines a quartic threefold in $\mathbb{C}^{4}$. As a consequence, the linear recurrence (3.12) holds for all $n$, and further implies the inhomogeneous linear equation

$$
x_{n+6}-\mathcal{J}_{1} x_{n+3}+x_{n}+\beta \mathcal{C}_{n}=0,
$$

where the quantity $\mathcal{C}_{n}$ varies with $n \bmod 3$. Writing everything in terms of coordinates

$$
\left(x_{0}, x_{1}, x_{2}, x_{3}\right) \in \mathbb{C}^{4}
$$

for the map (3.3) with $c=1$, this gives three independent quantities $\mathcal{C}_{j}$ given by (3.10) such that

$$
\left\{\mathcal{J}_{1}, \mathcal{C}_{j}\right\}_{1}=0, \quad j=0,1,2 \text {. }
$$

These $\mathcal{C}_{j}$ are not preserved by the map, but symmetric functions of them are, which produces the formulae (3.9) for three independent conserved quantities. The solution of the initial value problem can be conveniently expressed in the form (3.13), upon noting that (by separating out $n \bmod 3)$ the homogeneous form of (3.16) is equivalent to the second-order linear difference equation satisfied by the Chebyshev polynomials $T_{n}\left(\mathcal{J}_{1} / 2\right)=\cos (n \theta), U_{n}\left(\mathcal{J}_{1} / 2\right)=\sin (n \theta) / \sin \theta$ with $\mathcal{J}_{1}=2 \cos \theta$. 
Remark 3. The situation whereby an integrable system has more independent conserved quantities than the number of degrees of freedom is known as non-commutative integrability (in the sense of Nekhoroshev [37]), because not all these quantities can be in involution with one another. In this example, $\mathcal{J}_{1}$ Poisson commutes with both $\mathcal{J}_{2}$ and $\mathcal{J}_{3}$, but $\left\{\mathcal{J}_{2}, \mathcal{J}_{3}\right\}_{1} \neq 0$. The terminology 'superintegrable' is applied in the even more special situation that the number of independent integrals is one less than the dimension of the phase space [64], as is the case here.

Upon applying the Diophantine Laurentness Lemma to the case of initial data $(1,1,1,1)$, and choosing integer $\beta$ (with $\beta \neq 0$ to avoid the degenerate case of a fixed point) we get infinitely many solutions of certain Diophantine equations corresponding to the conserved quantities.

Corollary 2. With $\mathcal{J}_{1}=\mathcal{J}_{1}(p, q, r, s)$ as in (3.15),

$$
\begin{aligned}
& \mathcal{J}_{2}=\frac{p r^{2}(q+r+s)+s q^{2}(p+q+r)+p s(p r+p s+q s)+\beta q r(q+r)}{p q r s}, \\
& \mathcal{J}_{3}=\frac{\left(p s+q^{2}+r^{2}\right)\left(p r^{2}+p s^{2}+q^{2} s+\beta q r\right)\left(p r^{2}+p^{2} s+q^{2} s+\beta q r\right)}{p^{2} q^{2} r^{2} s^{2}},
\end{aligned}
$$

there are infinitely many integer solutions $(p, q, r, s)$ of the double pencil of Diophantine equations given by

$$
\lambda_{1} \mathcal{J}_{1}+\lambda_{2} \mathcal{J}_{2}+\lambda_{3} \mathcal{J}_{3}=\lambda_{1}\left(\beta^{2}+6 \beta+2\right)+\lambda_{2}(2 \beta+9)+3 \lambda_{3}(\beta+3)^{2},
$$

for all $\beta \in \mathbb{Z} \backslash\{0\}$, and any $\left(\lambda_{1}: \lambda_{2}: \lambda_{3}\right) \in \mathbb{P}^{2}$.

As was remarked after Proposition 2, the Laurent polynomials generated by the $c=0$ case of (3.2) show linear degree growth, so it might be anticipated that this case should also be linearizable. This indeed turns out to be so: the main results are very similar to the case $c=1$, and are stated below without proof.

Theorem 2. The map (3.3) for $c=0$ is superintegrable, in the sense that it has three independent conserved quantities $\tilde{\mathcal{J}}_{j}, j=1,2,3$, which satisfy

$$
\left\{\tilde{\mathcal{J}}_{1}, \tilde{\mathcal{J}}_{2}\right\}_{0}=0=\left\{\tilde{\mathcal{J}}_{1}, \tilde{\mathcal{J}}_{3}\right\}_{0},
$$

and also $\left\{\tilde{\mathcal{J}}_{1}, \mathcal{Q}_{j}\right\}_{0}=0$ for $j=0,1,2$, where

$$
\begin{aligned}
& \tilde{\mathcal{J}}_{1}=\mathcal{Q}_{0} \mathcal{Q}_{1} \mathcal{Q}_{2}-\mathcal{Q}_{0}-\mathcal{Q}_{1}-\mathcal{Q}_{2}, \quad \tilde{\mathcal{J}}_{2}=\mathcal{Q}_{0} \mathcal{Q}_{1}+\mathcal{Q}_{1} \mathcal{Q}_{2}+\mathcal{Q}_{2} \mathcal{Q}_{0}-3, \\
& \tilde{\mathcal{J}}_{3}=\mathcal{Q}_{0} \mathcal{Q}_{1} \mathcal{Q}_{2},
\end{aligned}
$$

with

$$
\mathcal{Q}_{0}=\frac{x_{0}+x_{2}}{x_{1}}, \quad \mathcal{Q}_{1}=\frac{x_{1}+x_{3}}{x_{2}}, \quad \mathcal{Q}_{2}=\frac{x_{1}}{x_{0}}+\frac{x_{2}}{x_{3}}+\frac{\beta}{x_{0} x_{3}} .
$$

The iterates of the corresponding recurrence

$$
x_{n+2} x_{n-2}=x_{n+1} x_{n-1}+\beta,
$$

satisfy the sixth-order linear recurrence

$$
x_{n+6}-\tilde{\mathcal{J}}_{1} x_{n+3}+x_{n}=0
$$

and the solution of the initial value problem for (3.21) can be written explicitly in terms of Chebyshev polynomials of the first and second kind ( $T_{n}$ and $U_{n}$ respectively), as

$$
x_{3 n+j}=\tilde{\mathcal{A}}_{j} T_{n}\left(\tilde{\mathcal{J}}_{1} / 2\right)+\tilde{\mathcal{B}}_{j} U_{n}\left(\tilde{\mathcal{J}}_{1} / 2\right), \quad j=0,1,2,
$$


where the coefficients $\tilde{\mathcal{A}}_{j}, \tilde{\mathcal{B}}_{j}$ are given by

$$
\tilde{\mathcal{A}}_{j}=2 x_{j}-\frac{2 x_{j+3}}{\tilde{\mathcal{J}}_{1}}, \quad \tilde{\mathcal{B}}_{j}=-x_{j}+\frac{2 x_{j+3}}{\tilde{\mathcal{J}}_{1}}
$$

for $j=0,1,2$.

We can apply the Diophantine Laurentness Lemma once more, taking initial data $(1,1,1,1)$ and $\beta \in \mathbb{Z}$, (with $\beta \neq 0,-1$ to avoid periodic orbits), to get infinitely many solutions of quartic Diophantine equations corresponding to the conserved quantities for (3.21).

Corollary 3. With the identification $(w, x, y, z)=\left(x_{n}, x_{n+1}, x_{n+2}, x_{n+3}\right)$, when $n=0$ the relations $(3.19)$ define $\tilde{\mathcal{J}}_{k}=\tilde{\mathcal{J}}_{k}(w, x, y, z)$ for $k=1,2,3$, as

$$
\begin{aligned}
& \tilde{\mathcal{J}}_{1}=\frac{\left(w^{2}+z^{2}\right) x y+\beta(w x+w z+y z)}{w x y z}, \\
& \tilde{\mathcal{J}}_{2}=\left(\begin{array}{c}
w^{2} z^{2}+x z\left(w^{2}+x^{2}+y^{2}+x z\right) \\
+w y\left(x^{2}+y^{2}+z^{2}+w y\right)+\beta\left(x^{2}+y^{2}+x z+w y\right)
\end{array}\right) /(w x y z), \\
& \tilde{\mathcal{J}}_{3}=\frac{(w+y)(x+z)(x z+y w+\beta)}{w x y z},
\end{aligned}
$$

and there are infinitely many integer solutions $(w, x, y, z)$ of the double pencil of Diophantine equations given by

$$
\lambda_{1} \tilde{\mathcal{J}}_{1}+\lambda_{2} \tilde{\mathcal{J}}_{2}+\lambda_{3} \tilde{\mathcal{J}}_{3}=\lambda_{1}(3 \beta+2)+\lambda_{2}(4 \beta+9)+4 \lambda_{3}(\beta+2),
$$

for all $\beta \in \mathbb{Z} \backslash\{0,-1\}$, and any $\left(\lambda_{1}: \lambda_{2}: \lambda_{3}\right) \in \mathbb{P}^{2}$.

Remark 4. The initial data $(1,1,1,1)$, together with the restrictions on $\beta$, are sufficient to ensure that the each of recurrences (3.11) and (3.21) generate non-periodic integer sequences, and hence infinitely many solutions of the corresponding Diophantine equations, given in Corollary 2 and Corollary 3 respectively. However, due to the fact that the recurrences are integrable (and even linearizable) in both cases, it is possible to choose much more general initial data and still generate integer sequences, which produce solutions of the same Diophantine equations but with different values on the right hand sides of (3.17) and (3.25) respectively.

\section{Outlook}

The Laurent property appears to be an extremely elegant, but somewhat special, feature of certain rational maps. In particular, it seems to hold for integrable bilinear or discrete Hirota type equations, such as (2.4) and (2.7), but also for the whole family (3.2), whose members have non-zero algebraic entropy for $c \geq 3$. For the latter family, we have noted the close connection between the Laurent property and the notion of singularity confinement as introduced in [19]. (For other examples of confined maps with the Laurent property see [29, 30].) This connection seems to persist for rational maps that do not themselves have the Laurent property.

For example, consider the second-order equation ${ }^{3}$

$$
u_{n+1}=\frac{\left(u_{n}\right)^{2}+1}{u_{n-1} u_{n}},
$$

which is superficially very similar to (2.13), and preserves the same symplectic form (2.15). The real phase portrait in $\mathbb{R}^{2}$ also looks qualitatively similar: Fig. 2 seems to display the same

\footnotetext{
${ }^{3}$ I am grateful to Vasilis Papageorgiou for showing me this example, which I believe is due to Claude Viallet.
} 
structure of invariant curves as Fig. 1. Furthermore, the equation (4.1) satisfies singularity confinement, with the singularity pattern being $\epsilon, \epsilon^{-1}, \epsilon^{-2}, \epsilon^{-1}, \epsilon$ (for $\left.\epsilon \rightarrow 0\right)$, which suggests making the substitution

$$
u_{n}=\frac{\tau_{n+2} \tau_{n-2}}{\tau_{n+1}\left(\tau_{n}\right)^{2} \tau_{n-1}} .
$$

Thus $u_{n}$ given as above satisfies (4.1) whenever $\tau_{n}$ satisfies

$$
\tau_{n+3} \tau_{n-3}=\left(\tau_{n+2} \tau_{n-2}\right)^{2}+\left(\tau_{n+1}\right)^{2}\left(\tau_{n}\right)^{4}\left(\tau_{n-1}\right)^{2},
$$

and this sixth-order recurrence has the Laurent property, as well as satisfying the singularity confinement test. The singularity pattern for (4.1), which includes poles, "unfolds" to yield isolated zeros, i.e. $\tau_{n}=\epsilon$ for some $n$ with adjacent iterates being $O(1)$ as $\epsilon \rightarrow 0$.

However, the logarithmic heights $h\left(\tau_{n}\right)$ of rational iterates grow exponentially with $n$. To see this, it is instructive to take all six initial values for (4.3) equal to 1, yielding the integer sequence

$$
1,1,1,1,1,1,2,5,29,1241,3642581,80305336110269, \ldots,
$$

which grows like $\log h\left(\tau_{n}\right)=\log \log \left|\tau_{n}\right| \sim n \log \gamma$ with $\log \gamma \approx 0.733$, where $\gamma \approx 2.081$ is the largest modulus root of the polynomial $\gamma^{4}-\gamma^{3}-2 \gamma^{2}-\gamma+1$. Hence the logarithmic heights of the rational numbers $u_{n}$ that lie in the orbit of $\left(u_{0}, u_{1}\right)=(1,1)$ generated by $(4.1)$ grow exponentially, and Halburd's Diophantine integrability criterion is failed. Similar arguments hold for generic orbits, and it follows that the curves appearing in Fig. 2 are not algebraic. To see this, recall that by the Hurwitz theorem a curve with an infinite order automorphism group has genus zero or one, and under iteration of such automorphisms the logarithmic heights of rational points grow linearly on a rational curve and quadratically on an elliptic one [48].

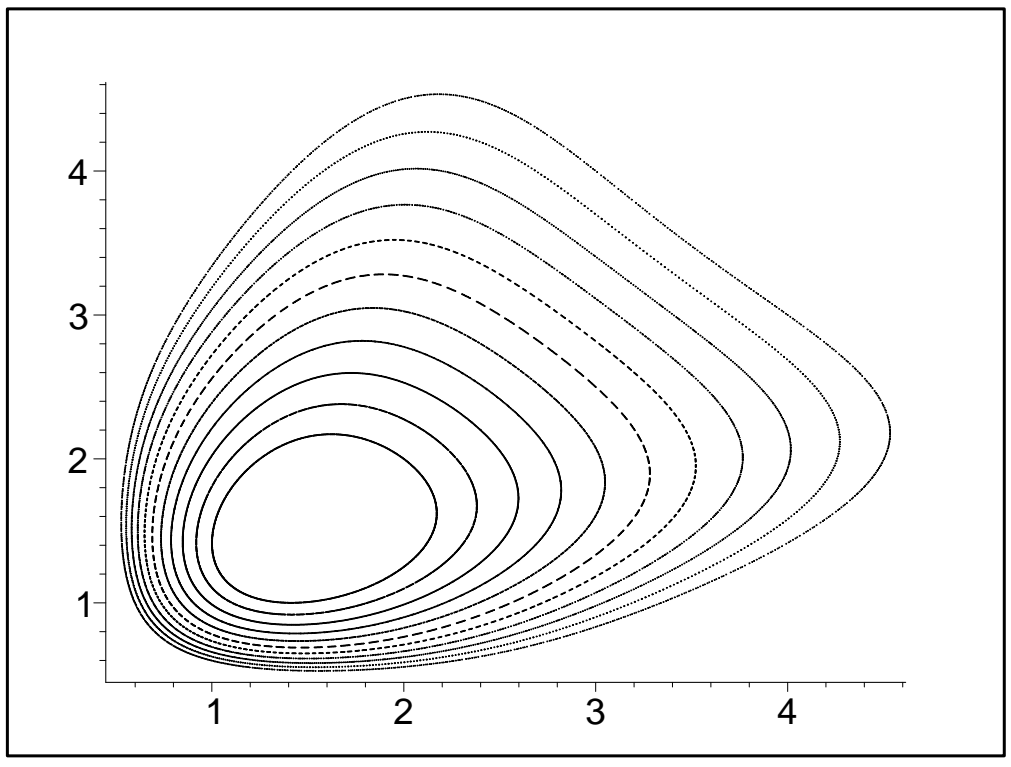

Figure 2. A family of orbits for the nonlinear recurrence (4.1).

We have concentrated on recurrences of the particular form (2.3), but this is not necessary for the Laurent property. Another interesting (and algebraically non-integrable) example is the second-order equation

$$
u_{n+1}+u_{n-1}=u_{n}+\frac{a}{\left(u_{n}\right)^{2}}, \quad a \neq 0,
$$


which in [21] was found by Hietarinta and Viallet to display singularity confinement with the pattern $\epsilon, \epsilon^{-2}, \epsilon^{-2}, \epsilon$, yet it has positive algebraic entropy and its real orbits display the characteristics of chaos. By way of the substitution

$$
u_{n}=\frac{\tau_{n+2} \tau_{n-1}}{\left(\tau_{n+1} \tau_{n}\right)^{2}}
$$

we arrive at the fifth-order recurrence

$$
\tau_{n+3}\left(\tau_{n}\right)^{3}\left(\tau_{n-1}\right)^{2}=\left(\tau_{n+2}\right)^{3}\left(\tau_{n-1}\right)^{3}-\left(\tau_{n+2}\right)^{2}\left(\tau_{n+1}\right)^{3} \tau_{n-2}+a\left(\tau_{n+1} \tau_{n}\right)^{6},
$$

which itself satisfies singularity confinement and has the Laurent property, i.e. for all $n$ the iterates satisfy $\tau_{n} \in \mathbb{Z}\left[\tau_{0}^{ \pm 1}, \ldots, \tau_{4}^{ \pm 1}, a\right]$. In this case, the logarithmic heights of rational iterates $\tau_{n} \in \mathbb{Q}$ (with $a \in \mathbb{Q}$ ) generically satisfy $h\left(\tau_{n}\right) \sim C \zeta^{n}$ for some $C>0$ and $\zeta=(3+\sqrt{5}) / 2$ is the square of the golden mean, while $\log \zeta$ turns out to be the value of the algebraic entropy for (4.4). Note that while the calculation of the algebraic entropy is quite involved [21, 55], it is quite straightforward to calculate the growth of heights from (4.6). Similarly to the previous example, the only confined singularities that appear in (4.6) are isolated zeros.

These examples illustrate the following general phenomenon: whenever we have a rational map with confined singularities, including poles, it should always be possible to "unfold" these into confined zeros, by embedding the map in higher dimensions via a change of variables, and the new map thus obtained should have the Laurent property. This is analogous to the way in which continuous integrable systems with the Painlevé property, that have meromorphic solutions, admit a Hirota bilinear form (or multilinear form) in terms of tau-functions that are holomorphic. Although this phenomenon (the existence of a tau-function) is very well known for discrete integrable systems [46], so far it does not seem to have been to have been exploited in the case of non-integrable maps.

\section{References}

[1] Braden H.W., Enolskii V.Z., Hone A.N.W., Bilinear recurrences and addition formulae for hyperelliptic sigma functions, J. Nonlinear Math. Phys. 12 (2005), suppl. 2, 46-62, math.NT/0501162.

[2] Bressoud D.M., Proofs and confirmations: the story of the alternating sign matrix conjecture, Cambridge University Press, Cambridge, 1999.

[3] Bruschi M., Ragnisco O., Santini P.M., Tu G.-Z., Integrable symplectic maps, Phys. D 49 (1991), $273-294$.

[4] Buchholz R.H., Rathbun R.L., An infinite set of heron triangles with two rational medians, Amer. Math. Monthly 104 (1997), 107-115.

[5] Buchstaber V.M., Enolskii V.Z., Leykin D.V., Hyperelliptic Kleinian functions and applications, in Solitons, Geometry and Topology: On the Crossroad, Editors V.M. Buchstaber and S.P. Novikov, AMS Translations Series 2, Vol. 179, AMS, 1997, 1-34, solv-int/9603005.

[6] Cantor D., On the analogue of the division polynomials for hyperelliptic curves, J. Reine Angew. Math. 447 (1994), 91-145.

[7] Carroll G., Speyer D., The cube recurrence, Electron. J. Combin. 11 (2004), \# R73, math.CO/0403417.

[8] Common A., Hone A.N.W., Musette M., A new discrete Hénon-Heiles system, J. Nonlinear Math. Phys. 10 (2003), suppl. 2, 27-40.

[9] Elaydi S., Discrete chaos, Chapman and Hall/CRC, Boca Raton, 2000.

[10] Einsiedler M., Everest G., Ward T., Primes in elliptic divisibility sequences, LMS J. Comput. Math. 4 (2001), 1-13.

[11] Everest G., Miller V., Stephens N., Primes generated by elliptic curves, Proc. Amer. Math. Soc. 132 (2003), 955-963.

[12] Everest G., van der Poorten A., Shparlinski I., Ward T., Recurrence sequences, AMS Mathematical Surveys and Monographs, Vol. 104, Amer. Math. Soc., Providence, RI, 2003. 
[13] Fedorov Y., Bäcklund transformations on coadjoint orbits of the loop algebra gl(r), J. Nonlinear Math. Phys. 9 (2002), suppl. 1, 29-46.

[14] Fomin S., Zelevinsky A., The Laurent phenomenon, Adv. Appl. Math. 28 (2002), 119-144, math.CO/0104241.

[15] Fomin S., Zelevinsky A., Cluster algebras IV: coefficients, Compos. Math., to appear, math.RA/0602259.

[16] Fordy A.P., Shabat A.B., Veselov A.P., Factorization and Poisson correspondences, Theor. Math. Phys. 105 (1995), 1369-1386.

[17] Gale D., The strange and surprising saga of the Somos sequences, Math. Intelligencer 13 (1991), no. 1, 40-42.

Gale D., Somos sequence update, Math. Intelligencer 13 (1991), no. 4, 49-50 (Reprinted in Tracking the Automatic Ant., Springer, 1998).

[18] Gekhtman M., Shapiro M., Vainshtein A., Cluster algebras and Poisson geometry, Moscow Math. J. 3 (2003), 899-934, math.QA/0208033.

[19] Grammaticos B., Ramani A., Papageorgiou V., Do integrable mappings have the Painlevé property? Phys. Rev. Lett. 67 (1991), 1825-1828.

[20] Halburd R.G., Diophantine integrability, J. Phys. A: Math. Gen. 38 (2005), L263-L269, nlin.SI/0504027.

[21] Hietarinta J., Viallet C., Singularity confinement and chaos in discrete systems, Phys. Rev. Lett. 81 (1998), 325-328, solv-int/9711014.

[22] Hone A.N.W., Non-autonomous Hénon-Heiles systems, Phys. D 118 (1998), 1-16, solv-int/9703005.

[23] Hone A.N.W., Kuznetsov V.B., Ragnisco O., Bäcklund transformations for the Hénon-Heiles and Garnier systems, CRM Proceedings and Lecture Notes, Vol. 25, Amer. Math. Soc., 2000, 231-235.

[24] Hone A.N.W., Kuznetsov V.B., Ragnisco O., Bäcklund transformations for many-body systems related to KdV, J. Phys. A: Math. Gen. 32 (1999), L299-L306, solv-int/9904003.

[25] Hone A.N.W., Kuznetsov V.B., Ragnisco O., Bäcklund transformations for the sl(2) Gaudin magnet, J. Phys. A: Math. Gen. 34 (2001), 2477-2490, nlin.SI/0007041.

[26] Hone A.N.W., Exact discretization of the Ermakov-Pinney equation, Phys. Lett. A 263 (1999), $347-354$.

[27] Hone A.N.W., Elliptic curves and quadratic recurrence sequences, Bull. Lond. Math. Soc. 37 (2005), 161171, Corrigendum, Bull. Lond. Math. Soc., 38 (2006), 741-742.

[28] Hone A.N.W., Sigma function solution of the initial value problem for Somos 5 sequences, Trans. Amer. Math. Soc., to appear, math.NT/0501554.

[29] Hone A.N.W., Diophantine non-integrability of a third-order recurrence with the Laurent property, J. Phys. A: Math. Gen. 39 (2006), L171-L177, math.NT/0601324.

[30] Hone A.N.W., Singularity confinement for maps with the Laurent property, Phys. Lett. A 361 (2007), 341-345, nlin.SI/0602007.

[31] Hone A.N.W., Discrete dynamics, integrability and integer sequences, Imperial College Press, in preparation.

[32] Kuznetsov V.B., Sklyanin E.K., On Bäcklund transformations for many-body systems, J. Phys. A: Math. Gen. 31 (1998), 2241-2251, solv-int/9711010.

[33] Kuznetsov V.B., Salerno M., Sklyanin E.K., Quantum Bäcklund transformation for the integrable DST model, J. Phys. A: Math. Gen. 33 (2000), 171-189, solv-int/9908002.

[34] Kuznetsov V.B., Vanhaecke P., Bäcklund transformations for finite-dimensional integrable systems: a geometric approach, J. Geom. Phys. 44 (2002), 1-40, nlin.SI/0004003.

[35] Kuznetsov V.B., Mangazeev V.V., Sklyanin E.K., Q-operator and factorised separation chain for Jack polynomials, Indag. Math. 14 (2003), 451-482, math.CA/0306242.

[36] Mills W.H., Robbins D.P., Rumsey H., Alternating-sign matrices and descending plane partitions, J. Combin. Theory Ser. A 34 (1983), 340-359.

[37] Nekhoroshev N.N., On action-angle variables and their generalizations, Tr. Moscow Math. Soc. 26 (1972), 181-198 (in Russian).

[38] Pasquier V., Gaudin M., The periodic Toda chain and a matrix generalization of the Bessel function recursion relations, J. Phys. A: Math. Gen. 25 (1992), 5243-5252.

[39] Kuznetsov V.B., Petrera M., Ragnisco O., Separation of variables and Bäcklund transformations for the symmetric Lagrange top, J. Phys. A: Math. Gen. 37 (2004), 8495-8512, nlin.SI/0403028. 
[40] Matsutani S., Recursion relation of hyperelliptic PSI-functions of genus two, Int. Transforms Spec. Func. 14 (2003), 517-527, math-ph/0105031.

[41] van der Poorten A.J., Elliptic curves and continued fractions, J. Integer Sequences 8 (2005), Article 05.2.5, 19 pages, math.NT/0403225.

[42] van der Poorten A.J., Swart C.S., Recurrence relations for elliptic sequences: every Somos 4 is a Somos $k$, Bull. Lond. Math. Soc. 38 (2006), 546-554, math.NT/0412293.

[43] Propp J., The many faces of alternating-sign matrices, Disc. Math. Theoret. Comp. Sci. Proc. AA (DMCCG) (2001), 43-58, math.CO/0208125.

[44] Propp J., The "bilinear" forum, http://www.math.wisc.edu propp/.

[45] Quispel G.R.W., Roberts J.A.G., Thompson C.J., Integrable mappings and soliton equations II, Phys. D 34 (1989), 183-192.

[46] Ramani A., Grammaticos B., Satsuma J., Bilinear discrete Painlevé equations, J. Phys. A: Math. Gen. 28 (1995), 4655-4665.

[47] Robinson R., Periodicity of Somos sequences, Proc. Amer. Math. Soc. 116 (1992), 613-619.

[48] Silverman J.H., The arithmetic of elliptic curves, Springer, 1986.

[49] Silverman J.H., p-adic properties of division polynomials and elliptic divisibility sequences, Math. Annal. 332 (2005), 443-471, Addendum, 473-474, math.NT/0404412.

[50] Sloane N.J.A., On-line encyclopedia of integer sequences, http://www.research.att.com/ njas $/$ sequences, sequence A006720.

[51] Speyer D., Perfect matchings and the octahedron recurrence, 2004, math.CO/0402452.

[52] Suris Y.B., The problem of integrable discretization: Hamiltonian approach, Progress in Mathematics, Vol. 219, Birkhäuser, Basel, 2003.

[53] Swart C.S., Elliptic curves and related sequences, PhD thesis, Royal Holloway, University of London, 2003.

[54] Swart C.S., Hone A.N.W., Integrality and the Laurent phenomenon for Somos 4 sequences, math.NT/0508094.

[55] Takenawa T., A geometric approach to singularity confinement and algebraic entropy, J. Phys. A: Math. Gen. 34 (2001), L95-L102, nlin.SI/0011037.

[56] Vanhaecke P., Integrable systems in the realm of algebraic geometry, 2nd ed., Springer, 2005.

[57] Veselov A.P., Integrable maps, Russ. Math. Surveys 46 (1991), 1-51.

[58] Veselov A.P., What is an integrable mapping? in What is Integrability? Editor V.E. Zakharov, SpringerVerlag, 1991, 251-272.

[59] Veselov A.P., Growth and integrability in the dynamics of mappings, Comm. Math. Phys. 145 (1992), 181-193.

[60] Veselov A.P., Shabat A.B., A dressing chain and the spectral theory of the Schrödinger operator, Funct. Anal. Appl. 27 (1993), 1-21.

[61] Ward M., Memoir on elliptic divisibility sequences, Amer. J. Math. 70 (1948), 31-74.

[62] Ward M., The law of repetition of primes in an elliptic divisibility sequence, Duke Math. J. 15 (1948), 941-946.

[63] Weiss J., Periodic fixed points of Bäcklund transformations and the Korteweg-de Vries equation, J. Math. Phys. 27 (1986), 2647-2656.

[64] Wojciechowski S., Superintegrability of the Calogero-Moser system, Phys. Lett. A 95 (1983), $279-281$.

[65] Yuzbashyan E.A., Kuznetsov V.B., Altshuler B.L., Integrable dynamics of coupled Fermi-Bose condensates, Phys. Rev. B $\mathbf{7 2}$ (2005), 144524, 9 pages, cond-mat/0506782.

[66] Yuzbashyan E.A., Altshuler B.L., Kuznetsov V.B., Enolskii V.Z., Nonequilibrium Cooper pairing in the nonadiabatic regime, Phys. Rev. B 72 (2005), 220503(R), 4 pages, cond-mat/0505493.

[67] Zagier D., Problems posed at the St. Andrews Colloquium, 1996, Solutions, 5th day, available at http://www-groups.dcs.st-and.ac.uk/ john/Zagier/Problems.html.

[68] Zabrodin A., A survey of Hirota's difference equations, Teor. Mat. Fiz. 113 (1997), 179-230 (in Russian), solv-int/9704001. 